\title{
Simplicial structures and normal forms for mapping class groups and braid groups
}

\author{
A J BERRICK \\ E HANBURY
}

\begin{abstract}
In this paper we show that the mapping class groups of any surface with nonempty boundary form a simplicial group as the number of marked points varies. This extends the simplicial structure on braid groups of surfaces found by Berrick, Cohen, Wong and $\mathrm{Wu}$. We use the simplicial maps to construct compatible normal forms for elements of the braid groups and mapping class groups of surfaces with boundary.
\end{abstract}

20F38; 20E22, 55U10

\section{Introduction}

Over the last decade, a number of results have revealed previously unsuspected relationships among mapping class groups of surfaces, their braid groups and homotopy theory. In particular, Berrick, Cohen, Wong and Wu [2] showed a linkage between the braid groups of the disc and sphere and homotopy groups of spheres. This was obtained by a study of the face maps between braid groups corresponding to the forgetting of strands in a braid. In [3; 4], the present authors, together with $\mathrm{J} \mathrm{Wu}$, showed that generalising such $\Delta$-structures both to braid groups on all surfaces and to their mapping class groups leads to more general relations involving homotopy theory.

Any discussion of $\Delta$-structures naturally prompts the question as to whether the face maps are part of a more comprehensive simplicial structure; that is, whether there are also degeneracy maps present. In the most familiar case, of pure braid groups on the disc, such a structure gives rise to semidirect products of the pure braid groups, and so the important Artin combing, from which one deduces solubility of the word problem in the braid groups. In this paper we explore the existence of degeneracies and normal forms.

For a surface $M$ (always connected, but not necessarily oriented or closed) and $k \geq 0$, recall that the configuration space

$$
\operatorname{Conf}_{k}(M)=\left\{\left(x_{0}, \ldots, x_{k-1}\right) \in M^{k} \mid x_{i} \neq x_{j} \text { when } i \neq j\right\},
$$


consists of all ordered configurations of $k$ points in $M$. Permuting the points gives a natural action of $\Sigma_{k}$ (the symmetric group on $k$ letters) on $\operatorname{Conf}_{k}(M)$; the resulting quotient space $\Sigma_{k} \backslash \operatorname{Conf}_{k}(M)$ is the space of all unordered configurations in $M$. The pure and full braid groups of $M$ are given respectively by

$$
\begin{aligned}
& P_{k}(M)=\pi_{1}\left(\operatorname{Conf}_{k}(M)\right), \\
& B_{k}(M)=\pi_{1}\left(\Sigma_{k} \backslash \operatorname{Conf}_{k}(M)\right) .
\end{aligned}
$$

Thus, elements of $P_{k}(M)$ are homotopy classes of loops in the configuration space, based at some chosen configuration, and elements of $B_{k}(M)$ can be considered as homotopy classes of paths in the configuration space that start at the chosen configuration and end at some permutation of it. There is a natural map $\pi: B_{k}(M) \rightarrow \Sigma_{k}$ given by recording the permutation of the chosen configuration at which a path ends. Evidently $\operatorname{Ker}(\pi)=P_{k}(M)$.

In [2] the authors study the sequences

$$
\begin{aligned}
& \underline{B}(M)=\left\{B_{1}(M), B_{2}(M), \ldots\right\}, \\
& \underline{P}(M)=\left\{P_{1}(M), P_{2}(M), \ldots\right\} .
\end{aligned}
$$

They describe functions $d_{i}: B_{k+1}(M) \rightarrow B_{k}(M), 0 \leq i \leq k$, known as face maps, given by deleting the $i^{\text {th }}$ strand of a braid. These are not group homomorphisms but they satisfy the crossed identity $d_{i}\left(\beta_{1} \beta_{2}\right)=d_{i}\left(\beta_{1}\right) d_{i \cdot \pi\left(\beta_{1}\right)}\left(\beta_{2}\right)$ for all $\beta_{1}, \beta_{2} \in B_{k+1}(M)$. Here $i \cdot \pi\left(\beta_{1}\right)$ denotes the result of performing the permutation $\pi\left(\beta_{1}\right)$ on $i$. Since $\pi$ is the identity on the pure braid group, then $d_{i}$ restricts to a group homomorphism $d_{i}: P_{k+1}(M) \rightarrow P_{k}(M)$.

In the case that $M$ admits a nonvanishing vector field (equivalently, $M$ has nonempty boundary or zero Euler characteristic) there are also maps in the other direction, the socalled degeneracy maps $s_{j}: B_{k}(M) \rightarrow B_{k+1}(M)$. The map $s_{j}$ is given by "doubling up" the $j^{\text {th }}$ strand of a braid; that is, we take another copy of the path in the $j^{\text {th }}$ component and use the vector field to translate it a small distance from the original. The nonvanishing vector field enables us to do this in a coherent fashion. Again, the functions $s_{j}$ are not group homomorphisms but they do satisfy a crossed identity analogous to the one above and when we restrict to the pure braid groups, we get a group homomorphism.

The face maps and degeneracy maps just described satisfy the so-called simplicial identities. Thus, for surfaces $M$ admitting a nonvanishing vector field, $\underline{B}(M)$ is a crossed simplicial group and $\underline{P}(M)$ is a simplicial group. Simplicial groups can be considered as combinatorial models of spaces; any simplicial group $\underline{G}$ has an associated sequence of homotopy sets $\pi_{n}(\underline{G})$. One of the main results of [2] was that 
each $\pi_{n}\left(\underline{P}\left(S^{2}\right)\right)$ is a group and there are isomorphisms $\pi_{n}\left(\underline{P}\left(S^{2}\right)\right) \cong \pi_{n}\left(S^{2}\right)$ for all $n \geq 4$. This gives a new description and a possible new method of calculation for the homotopy groups of spheres.

The braid groups $P_{k}(M)$ and $B_{k}(M)$ are closely related to (and indeed, with the exception of a few low degree cases, are subgroups of) the important mapping class groups $\Gamma^{k}(M)$ and $\Gamma^{(k)}(M)$. We have

$$
\Gamma^{(k)}(M)=\pi_{0}\left(\operatorname{Diff}\left(M,\left[\boldsymbol{m}_{k}\right]\right)\right),
$$

where $\operatorname{Diff}\left(M,\left[\boldsymbol{m}_{k}\right]\right)$ is the group of self-diffeomorphisms of $M$ that leave invariant a set $\left[\boldsymbol{m}_{k}\right]$ of $k$ marked points. If $M$ has nonempty boundary, the diffeomorphisms are required to fix it pointwise, and if $M$ is orientable the diffeomorphisms are assumed to be orientation-preserving. The group $\Gamma^{k}(M) \leq \Gamma^{(k)}(M)$ is the subgroup corresponding to those diffeomorphisms that also fix each of the marked points. These definitions also make sense when $k=0$, in which case we denote the mapping class group by $\Gamma(M)$.

In [4] it was shown that for any surface $M$, the face maps on the braid groups of $M$ extend to maps $d_{i}: \Gamma^{(k+1)}(M) \rightarrow \Gamma^{(k)}(M)$. Geometrically, the map $d_{i}$ can be described as forgetting the $i^{\text {th }}$ marked point. Again, it is not a group homomorphism, but it satisfies a crossed identity and when we restrict to $\Gamma^{k+1}(M)$, we get a group homomorphism.

For the sequence $\underline{\Gamma}(M)=\left\{\Gamma^{1}(M), \Gamma^{2}(M), \ldots\right\}$, the existence of face homomorphisms makes it possible to define homotopy sets $\pi_{n}(\underline{\Gamma}(M))$. Thus, one of the main results of [4] was that the homotopy sets of $\underline{\Gamma}(M)$ are isomorphic to those of $\underline{P}(M)$, except in a few low-degree cases.

We extend the results of $[2 ; 4]$ by considering degeneracy maps on mapping class groups. We show that for surfaces with nonempty boundary, it is possible to extend the degeneracy maps for braid groups to maps of mapping class groups. We do this by considering splittings

$$
\Gamma^{(k)}(M) \cong \Gamma(M) \ltimes B_{k}(M) \text { and } \Gamma^{k}(M) \cong \Gamma(M) \ltimes P_{k}(M)
$$

which hold for all surfaces $M$ with nonempty boundary. Our main result is the following.

Theorem A For a surface $M$ with nonempty boundary, $\underline{\Gamma}^{\mathrm{sym}}(M)$ forms a crossed simplicial group and $\underline{\Gamma}(M)$ forms a simplicial group. These simplicial structures extend the known simplicial structures for braid groups. 
$\mathrm{Li}$ and $\mathrm{Wu}[10]$ used the crossed simplicial group structure on the classical braid groups (on the disc) to show that the centre of $B_{k}=B_{k}\left(D^{2}\right)$ modulo the boundary Brunnian braids is $\mathbb{Z}$ times the group of exponent 2 elements in $\pi_{k}\left(S^{2}\right)$. On the other hand, the centre of its associated pure braid group $P_{k}$ modulo the boundary Brunnian braids is $\mathbb{Z} \times \pi_{k}\left(S^{2}\right)$, as reflected in the conjugation action of $B_{k}$ and $P_{k}$. A similar situation occurs in $\mathrm{Wu}$ [11], where the crossed simplicial group structure is essentially used for distinguishing the actions of $B_{k}$ and $P_{k}$ via the Artin representation on the group whose centre is the homotopy group. Accordingly, the crossed simplicial group structure of the mapping class group might aid understanding of its conjugation action on Brunnian mapping classes.

As pointed out by the referee, there is a possible general categorical application of crossed simplicial groups. One can consider a crossed simplicial group $\left\{G_{k}\right\}_{k \geq 0}$ as a category $\mathcal{G}$ where the morphisms are endomorphisms of the $G_{k}$ generated by group translations and face and degeneracy operations. In the case of the crossed simplicial group of finite cyclic groups, functors to an abelian category give rise to cyclic homology theory; other crossed simplicial groups lead to their own associated homology theories; see Fiedorowicz and Loday [8].

We use the simplicial structures on braid groups and mapping class groups to construct semidirect product decompositions for these groups and normal forms for their elements. In Section 3 we show that $P_{k+1}(M)$ is an iterated semidirect product of $\pi_{1}(M)$ and $k$ finitely generated free groups, and this allows us to read off the following normal form for braids.

Theorem B Let $M$ be a surface admitting a nonvanishing vector field. For $k \geq 0$, every element of $P_{k+1}(M)$ can be written in a unique way as $\alpha_{0} \cdots \alpha_{k}$, where $\alpha_{i}$ is represented by a path in $\operatorname{Conf}_{k+1}(M) \subseteq M^{k+1}$ in which the first $i$ points are stationary and the remaining points move in parallel (with respect to the vector field), tracing a path in $M$.

By considering the decomposition $\Gamma^{k+1}(M) \cong \Gamma(M) \ltimes P_{k+1}(M)$, we get the following normal form for mapping classes.

Theorem $\mathbf{C}$ Let $M$ be a surface with boundary. For $k \geq 0$, every element of $\Gamma^{k+1}(M)$ can be written in a unique way as $\theta \phi_{0} \cdots \phi_{k}$, where $\theta$ is the identity on a collar neighbourhood of a boundary component that contains the marked points and $\phi_{i}$ is given by pushing the marked points around some $\alpha_{i}$ as in Theorem $B$.

Alternative normal forms, analogous to Artin combing for braids over the disc, are given in Section 3. Such forms derive from coface maps rather than simplicial maps, where 
coface maps are an aspect of the bi- $\Delta$-group structure studied in $\mathrm{Wu}$ [12]. This suggests interesting speculations on the relationships between simplicial group structures and bi$\Delta$-group structures that enrich the same $\Delta$-group structure. Developing a theme that appears to originate in Cohen [5], $\mathrm{Wu}$ [12, Example 1.2.8] relates results in homotopy theory concerning Hopf invariants to the theory of braids. In particular, one can use normal forms on braids to determine the Cohen braids; see Bardakov, Mikhailov, Vershinin and $\mathrm{Wu}[1]$.

The paper is organised as follows. In Section 2 we recall the simplicial structure on the symmetric groups and the definition of a crossed simplicial group. We review the simplicial group structure on the braid groups, as given in [2], and establish a preferred set of generators for braids. Finally, we explain how to extend the degeneracy maps to the mapping class groups, in the case of surfaces with nonempty boundary. In Section 3 we look at how these algebraic structures can be used to obtain semidirect product decompositions for braid groups and mapping class groups of surfaces and we display normal forms for elements of these groups.

Acknowledgements The authors are pleased to acknowledge the benefit of conversations with Jie $\mathrm{Wu}$ and Luis Paris at the early stages of preparing this paper, and are grateful for the support of NUS research grants R-146-000-137-112 and R-146-000143-112. Comments of an anonymous referee have also enhanced the discussion.

\section{Simplicial structures}

A simplicial set is a sequence of sets $\underline{X}=\left\{X_{0}, X_{1}, \ldots\right\}$ together with face maps $d_{i}: X_{k} \rightarrow X_{k-1}$ and degeneracy maps $s_{j}: X_{k} \rightarrow X_{k+1}$ for each $i, j=0, \ldots, k$. The face maps and degeneracy maps must satisfy the simplicial identities

$$
\begin{aligned}
& d_{j} d_{i}=d_{i} d_{j+1} \quad \text { whenever } i \leq j, \\
& s_{i} s_{j}=s_{j+1} s_{i} \quad \text { whenever } i \leq j, \\
& d_{i} s_{j}= \begin{cases}s_{j-1} d_{i} & \text { whenever } i<j, \\
\mathrm{id} & \text { whenever } i=j \text { or } j+1, \\
s_{j} d_{i-1} & \text { whenever } i>j+1 .\end{cases}
\end{aligned}
$$

If each $X_{k}$ is a group and the face maps and degeneracy maps are group homomorphisms, then $\underline{X}$ is said to be a simplicial group. A sequence of sets equipped with only face maps (that is, no degeneracy maps) is called a $\Delta$-set. Similarly we have the notion of $\Delta$-group. 


\subsection{Simplicial structure on the symmetric groups}

The collection of symmetric groups $\underline{\Sigma}=\left\{\Sigma_{k+1}\right\}_{k \geq 0}$ is an important example of a simplicial set. We regard $\Sigma_{k}$ as the group of permutations of $[k]=\{0, \ldots, k-1\}$ and write $i \cdot \sigma$ for the result of evaluating $\sigma$ on $i$. Thus $\sigma \tau$ denotes the permutation obtained by first applying $\sigma$ and then $\tau$.

The face maps $d_{i}: \Sigma_{k} \rightarrow \Sigma_{k-1}(i=0,1, \ldots, k-1)$ are defined by commutative diagrams



where $d^{i}:[n-1] \rightarrow[n]$ is the unique order-preserving map that misses $i$. If we represent a permutation by a diagram of arrows as in Figure 1, then $d_{i}$ removes the arrow that starts at $i$.

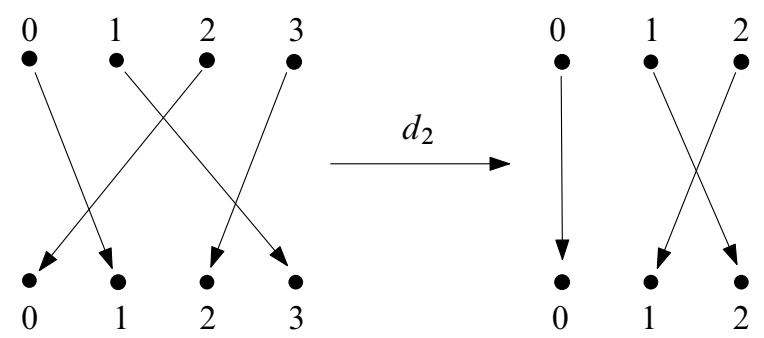

Figure 1: The face maps for the symmetric groups

On the other hand, we can define a degeneracy map $s_{j}: \Sigma_{k} \rightarrow \Sigma_{k+1}(j=0,1, \ldots, k-1)$ by doubling up the arrow that starts at $j$; see Figure 2 . This can be defined algebraically by requiring that

$$
j \cdot s_{j}(\sigma)=j \cdot \sigma, \quad(j+1) \cdot s_{j}(\sigma)=j \cdot \sigma+1
$$

and that the following diagram commutes:

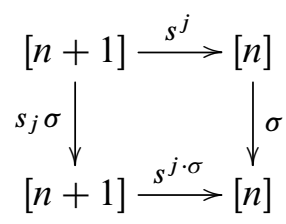

Here $s^{j}:[n+1] \rightarrow[n]$ is the unique order preserving map that hits $j$ twice. It can easily be checked that the simplicial identities hold. 


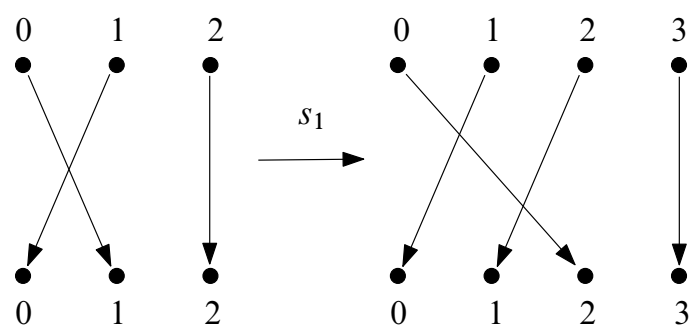

Figure 2: The degeneracy maps for the symmetric groups

\subsection{Definition of a crossed simplicial group}

There is an intermediate notion between simplicial set and simplicial group, that of a crossed simplicial group. This is a simplicial set $\underline{X}=\left\{X_{k}\right\}_{k \geq 0}$ for which each $X_{i}$ is a group and there are homomorphisms $\pi: X_{k} \rightarrow \Sigma_{k+1}$ that commute with the face and degeneracy maps and satisfy

$$
\begin{aligned}
& d_{i}\left(x_{1} x_{2}\right)=d_{i}\left(x_{1}\right) d_{i \cdot \pi\left(x_{1}\right)}\left(x_{2}\right), \\
& s_{j}\left(x_{1} x_{2}\right)=s_{j}\left(x_{1}\right) s_{j \cdot \pi\left(x_{1}\right)}\left(x_{2}\right) .
\end{aligned}
$$

(Note that there are several choices to be made with regards to which side the symmetric group acts on $\{0,1, \ldots, k-1\}$ and how the face maps for symmetric groups are defined. Different choices give rise to different versions of these identities.)

The symmetric groups with $\pi$ as the identity map form the prototypical crossed simplicial group. If $\underline{X}$ is any other crossed simplicial group then the homomorphisms $\pi: X_{k} \rightarrow \Sigma_{k+1}$ fit together to give a morphism of crossed simplicial groups $\pi: \underline{X} \rightarrow \underline{\Sigma}$, that is, a collection of maps respecting the crossed simplicial group structure.

Any simplicial group is a crossed simplicial group with $\pi$ taken to be the trivial homomorphism. In the converse direction, a crossed simplicial group need not be a simplicial group. There is a corresponding notion of crossed $\Delta$-group when we have only the face maps.

A useful fact about crossed simplicial groups is as follows.

Lemma 2.1 If $\underline{X}$ is a crossed simplicial (resp. $\Delta-$ ) group and $\pi: \underline{X} \rightarrow \underline{\Sigma}$ is the associated morphism, then $\operatorname{Ker} \pi \leq \underline{X}$ is a simplicial (resp. $\Delta_{-}$) group.

\subsection{Simplicial structure on braid groups}

Let $M$ be any surface. Select marked points $m_{0}, m_{1}, \ldots$ in $M$. We write $\boldsymbol{m}_{k}$ for the ordered set of marked points $\left(m_{0}, \ldots, m_{k-1}\right)$ and $\left[\boldsymbol{m}_{k}\right]$ for the corresponding unordered set of points. 
To define face maps and degeneracy maps for the braid groups, we think of $B_{k}(M)$ as the set of path-homotopy classes $[\lambda]$ of paths $\lambda$ in $\operatorname{Conf}_{k}(M)$ that start at the distinguished configuration $\boldsymbol{m}_{k}$ and end at some permutation of it. To describe the multiplication on $B_{k}(M)$, we use the map

$$
\pi: B_{k}(M) \rightarrow \Sigma_{k}
$$

that sends $[\lambda] \in B_{k}(M)$ to the permutation $\pi[\lambda]$ defined by

$$
\lambda(1)=\left(m_{0 \cdot \pi[\lambda]}, \ldots, m_{(k-1) \cdot \pi[\lambda]}\right) .
$$

For $[\lambda] \in B_{k}(M)$, let $\left(\lambda^{0}, \ldots, \lambda^{k-1}\right)$ be the coordinates of $\lambda$. There is a left action of $\Sigma_{k}$ on $\operatorname{Conf}_{k}(M)$ given by

$$
\sigma \cdot\left(x_{0}, \ldots, x_{k-1}\right)=\left(x_{0 \cdot \sigma}, \ldots, x_{(k-1) \cdot \sigma}\right)
$$

and this induces a left action of $\Sigma_{k}$ on $B_{k}(M)$ given by

$$
\sigma \cdot[\lambda]=[\sigma \cdot \lambda]
$$

where $\sigma \cdot \lambda$ denotes the path $\left(\lambda^{0 \cdot \sigma}, \ldots, \lambda^{(k-1) \cdot \sigma}\right)$ in $\operatorname{Conf}_{k}(M)$. Now the product in $B_{k}(M)$ is given by

$$
\left[\lambda_{1}\right]\left[\lambda_{2}\right]=\left[\lambda_{1} *\left(\pi\left[\lambda_{1}\right] \cdot \lambda_{2}\right)\right],
$$

where $*$ denotes the usual concatenation product of paths (read from left to right). With this multiplication, the map $\pi$ is a group homomorphism. The kernel of $\pi$ is $P_{k}(M)=\pi_{1}\left(\operatorname{Conf}_{k}(M), \boldsymbol{m}_{k}\right)$, the $k^{\text {th }}$ pure braid group of $M$.

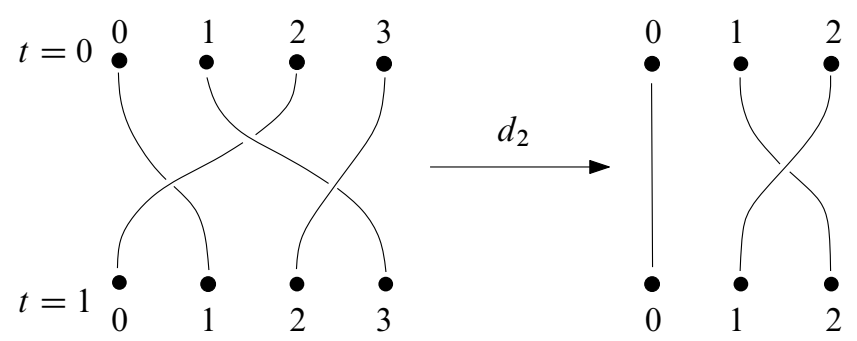

Figure 3: The face maps for the braid groups

In [2] it is shown that for any surface $M$, the braid groups of $M$ form a crossed $\Delta$-group $\underline{B}(M)$ with

$$
\underline{B}(M)_{k}=B_{k+1}(M) .
$$

The homomorphism $\underline{B}(M)_{k} \rightarrow \Sigma_{k+1}$ is provided by the homomorphism $\pi$ defined above. The face map $d_{i}: B_{k+1}(M) \rightarrow B_{k}(M)$ removes the $i^{\text {th }}$ component of a path in $\operatorname{Conf}_{k+1}(M)$. Geometrically, we think of $d_{i}$ as removing the $i^{\text {th }}$ strand of a braid, 
as in Figure 3. A slight modification to the endpoints is necessary so that the resulting path represents an element of $B_{k}(M)$; see [4, Section 2.3] for details.

In the case that $M$ admits a nonvanishing vector field, there are also degeneracy maps for $\underline{B}(M)$, defined using the vector field. The idea is that the degeneracy map $s_{j}: B_{k}(M) \rightarrow B_{k+1}(M)$ is given by doubling up the $j^{\text {th }}$ string in a braid; see Figure 4.

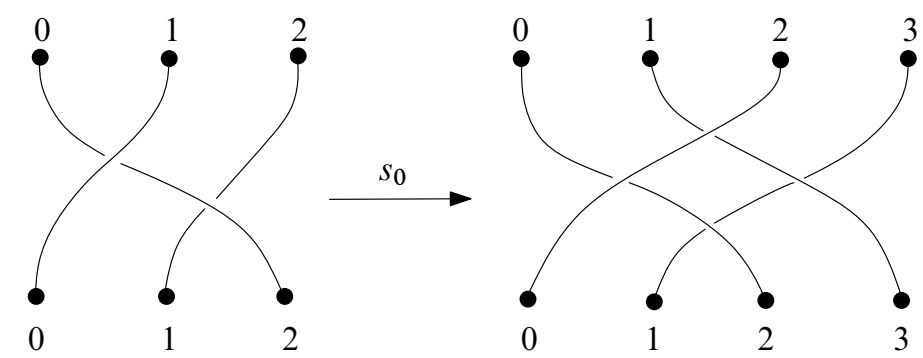

Figure 4: The degeneracy maps for the braid groups

This can be described explicitly on the configuration space level by sending a path $\left(\lambda^{0}, \ldots, \lambda^{k-1}\right)$ in $\operatorname{Conf}_{k}(M)$ to

$$
\left(\lambda^{0}, \ldots, \lambda^{j}, \lambda^{j}+\epsilon, \ldots, \lambda^{k-1}\right)
$$

where $\lambda^{j}+\epsilon$ is obtained by sliding $\lambda^{j}$ a small distance in the direction of the vector field. Again, a small modification regarding the basepoints is necessary to make this properly defined; for full details see [2, Section 3.2].

Now recall that the kernel of $\pi: \underline{B}(M) \rightarrow \underline{\Sigma}$ is $\underline{P}(M)$ where $\underline{P}(M)_{k}=P_{k+1}(M)$. The situation as to when $\underline{B}(M)$ and $\underline{P}(M)$ admit simplicial structures may be summarised as follows.

Theorem 2.2 Let $M$ be an arbitrary surface.

(a) The collection $\underline{B}(M)=\left\{B_{k+1}(M)\right\}_{k \geq 0}$ forms a crossed $\Delta$-group, and

$$
\underline{P}(M)=\left\{P_{k+1}(M)\right\}_{k \geq 0}
$$

forms a $\Delta$-group.

(b) The following are equivalent:

(i) $\underline{B}(M)$ is a crossed simplicial group.

(ii) $\underline{P}(M)$ is a simplicial group.

(iii) $M$ admits a nonvanishing vector field. 
Proof Part (a) is simply [2, Proposition 4.2.1 (1), (2)]. For (b), [2, Corollary 3.2.7, Theorem 3.2.12] show that (iii) $\Rightarrow$ (i), while (i) $\Rightarrow$ (ii) by Lemma 2.1 above. It remains to show that the existence of a nonvanishing vector field is also a necessary condition for the existence of degeneracies. For surfaces other than $S^{2}$, this follows from the fact (see Gonçalves and Guaschi [9, Theorem 2]) that there is no section of $d_{k}: P_{k+1}(M) \rightarrow P_{k}(M)$ when $k \geq 3$ (by means of the simplicial identities in Section 2 above). For $S^{2}$, further analysis [2, Proposition 3.2.14] gives the result.

To conclude this section, we explain how the face and degeneracy maps can be used to construct preferred generating sets for $P_{k}(M)$ and $B_{k}(M)$. We begin with a generating set $\mathcal{X}$ for $\pi_{1}(M)$. First, fix a basepoint $x_{0} \in M$ away from all the marked points $m_{i}$, and take representatives for the elements of $\mathcal{X}$ that are loops based at $x_{0}$ that avoid all points $m_{i}$. Now, for each $i \geq 0$, let us choose a generating set $\mathcal{X}^{i}$ for $\pi_{1}\left(M-\boldsymbol{m}_{i}, m_{i}\right)$. If $i=0, \boldsymbol{m}_{i}$ is assumed to be empty; the set $\mathcal{X}^{0}$ is obtained by conjugating elements of $\mathcal{X}$ by a path from $m_{0}$ to $x_{0}$. If $i \geq 1$, the set $\mathcal{X}^{i}$ consists of loops of the following two types. Those of the first type are given by concatenating a fixed path $\delta$ from $m_{i}$ to $x_{0}$, a generator in $\mathcal{X}$ for $\pi_{1}\left(M, x_{0}\right)$ and $\delta^{-1}$. The second type are small, simple loops, based at $m_{i}$, each of which encircles one of the marked points $m_{0}, \ldots, m_{i-1}$. See Figure 5 .



Figure 5: Generating set $\mathcal{X}^{i}$ for $\pi_{1}\left(M-\boldsymbol{m}_{i}, m_{i}\right)$

Next, recall from [3, Lemma 3.11] that for each $j \geq 0$ the map

$$
\begin{aligned}
M-\boldsymbol{m}_{j} & \longrightarrow \operatorname{Conf}_{j+1}(M), \\
x & \longmapsto\left(m_{0}, \ldots, m_{j-1}, x\right),
\end{aligned}
$$


induces the injection $\iota_{j}$ in the short exact sequence

$$
1 \longrightarrow \pi_{1}\left(M-\boldsymbol{m}_{j}, m_{j}\right) \stackrel{\iota_{j}}{\longrightarrow} P_{j+1}(M) \stackrel{d_{j}}{\longrightarrow} P_{j}(M) \longrightarrow 1 .
$$

Let $c_{m_{i}}$ denote a constant path at $m_{i}$. On the group level, $\iota_{j}$ sends a loop $\gamma$ in $M-\boldsymbol{m}_{j}$ to the loop $\left(c_{m_{0}}, \ldots, c_{m_{j-1}}, \gamma\right)$ in the configuration space. We take $\operatorname{Conf}_{0}(M)$ to be a point so that $P_{0}(M)$ is trivial. In the case $j=0, \iota_{j}$ is the identity map from $\pi_{1}\left(M, m_{0}\right)$ to $P_{1}(M)$.

For $0 \leq i \leq k-2$, define

$$
\mathcal{Y}_{k}^{i}=s_{k-2} \circ \cdots \circ s_{i} \circ \iota_{i}\left(\mathcal{X}^{i}\right) \subseteq P_{k}(M),
$$

while $\mathcal{Y}_{k}^{k-1}=\iota_{k-1}\left(\mathcal{X}^{k-1}\right) \subseteq P_{k}(M)$. By considering the action of $\iota$ and $s$, we can see that every element of $\mathcal{Y}_{k}^{i}$ is a path in $\operatorname{Conf}_{k}(M)$ of the form

$$
\left(c_{m_{0}}, \ldots, c_{m_{i-1}}, \gamma, \gamma+\epsilon, \ldots, \gamma+(k-1) \epsilon\right),
$$

where $\gamma \in \mathcal{X}^{i}$. Thus, elements of $\mathcal{Y}_{k}^{i}$ are represented by paths in which the first $i$ points remain stationary and the remaining $k-i$ points move "in parallel", tracing an element of $\mathcal{X}^{i}$.

Lemma 2.3 For any surface $M$ that admits a nonvanishing vector field, the set $\mathcal{Y}_{k}=\bigcup_{i=0}^{k-1} \mathcal{Y}_{k}^{i}$ is a finite generating set for $P_{k}(M)$.

Proof The result certainly holds for $k=1$ when $P_{k}(M)=\pi_{1}\left(M, m_{0}\right)$ and $\mathcal{Y}_{k}=$ $\mathcal{Y}_{1}^{0}=\mathcal{X}^{0}$. Using the exact sequence (5) with $k$ substituted for $j$, we proceed by induction on $k$. Suppose that $k \geq 1$.

First observe that because $d_{k}: P_{k+1}(M) \rightarrow P_{k}(M)$ has $d_{k} \circ s_{k-1}=\operatorname{id}_{P_{k}(M)}$, we have

$$
d_{k}\left(\mathcal{Y}_{k+1}^{i}\right)=\mathcal{Y}_{k}^{i} \quad \text { for each } i \in\{0, \ldots, k\} .
$$

Thus, by the induction hypothesis, $d_{k}\left(\bigcup_{i=0}^{k-1} \mathcal{Y}_{k+1}^{i}\right)=\mathcal{Y}_{k}$ generates $P_{k}(M)$. Finally, since $\mathcal{Y}_{k+1}^{k}=\iota_{k}\left(\mathcal{X}^{k}\right)$ generates $\operatorname{Im} \iota_{k}=\operatorname{Ker} d_{k}$, the set $\mathcal{Y}_{k+1}$ does after all generate $P_{k+1}(M)$.

Let $\mathcal{S}_{k} \subseteq B_{k}(M)$ be a set of elements such that $\pi\left(\mathcal{S}_{k}\right)$ generates $\Sigma_{k}$. We may assume that the paths representing the elements of $\mathcal{S}_{k}$ are contained in a small neighbourhood of the marked points.

Lemma 2.4 For any surface $M$ that admits a nonvanishing vector field, the set $\mathcal{S}_{k} \cup \mathcal{Y}_{k}$ is a finite generating set for $B_{k}(M)$. 
Proof This is immediate from Lemma 2.3 since $B_{k}(M)$ fits into the short exact sequence

$$
1 \longrightarrow P_{k}(M) \longrightarrow B_{k}(M) \stackrel{\pi}{\longrightarrow} \Sigma_{k} \longrightarrow 1 \text {. }
$$

\subsection{Simplicial structure on mapping class groups}

We use $x \cdot \phi$ (respectively $\boldsymbol{x} \cdot \phi$ ) to denote the result of evaluating a diffeomorphism $\phi$ on the point $x \in M$ (resp. $\left.\boldsymbol{x} \in \operatorname{Conf}_{k+1}(M)\right)$ and $\phi \odot \psi$ to denote the diffeomorphism given by first performing $\phi$ and then $\psi$. The right action of the diffeomorphism group on the surface is thereby compatible with our other choices of actions.

In [4], it is shown that $\underline{\Gamma}^{\mathrm{sym}}(M)$ defined by

$$
\underline{\Gamma}^{\mathrm{sym}}(M)_{k}=\Gamma^{(k+1)}(M)
$$

is a crossed $\Delta$-group and that $\underline{\Gamma}(M)$ given by

$$
\underline{\Gamma}(M)_{k}=\Gamma^{k+1}(M)
$$

is a $\Delta$-group. The face map $d_{i}: \Gamma^{(k+1)}(M) \rightarrow \Gamma^{(k)}(M)$ is given by forgetting the $i^{\text {th }}$ marked point, and similarly for $d_{i}: \Gamma^{k+1}(M) \rightarrow \Gamma^{k}(M)$. This definition must be modified slightly to ensure that for a mapping class $\phi$, both $d_{i} \phi$ and $d_{j} \phi$ fix the same set of marked points; see [4, Section 2.4] for details. The map to the symmetric group $\mu: \Gamma^{(k+1)}(M) \rightarrow \Sigma_{k+1}$ is defined by

$$
\left(m_{0}, \ldots, m_{k}\right) \cdot \psi=\left(m_{0 \cdot \mu(\psi)}, \ldots, m_{k \cdot \mu(\psi)}\right)
$$

for $[\psi] \in \Gamma^{(k+1)}(M)$. Thus, $\mu$ records how the diffeomorphisms permute the marked points. Full details are given in [4, Section 2.4].

In this section we deal with surfaces with nonempty boundary; we extend the degeneracy maps for braid groups to give degeneracies for mapping class groups and thus prove that $\underline{\Gamma}^{\mathrm{sym}}(M)$ (resp. $\left.\underline{\Gamma}(M)\right)$ is in fact a crossed simplicial group (resp. simplicial group).

In what follows we make use of the so-called push-map $p: B_{k}(M) \rightarrow \Gamma^{(k)}(M)$; see for example Farb and Margalit [7, Section 4.2]. For a path $\lambda=\left(\lambda^{0}, \ldots, \lambda^{k-1}\right)$ in the configuration space, define $p([\lambda])=\left[\Psi_{\lambda}(1)\right]$, where $\Psi_{\lambda}$ is the diffeotopy of $M$ given by simultaneously pushing a small disc containing $m_{i}$ around the path $\lambda^{i}$ for $i=0, \ldots, k-1$ (this extends the homomorphism $\partial^{P}: P_{k}(M) \rightarrow \Gamma^{k}(M)$ given in $[4$, Section 3]). 
Lemma 2.5 For all surfaces $M$ with boundary and for all $k \geq 1$, there are split short exact sequences

$$
\begin{aligned}
& 1 \longrightarrow B_{k}(M) \stackrel{p}{\longrightarrow} \Gamma^{(k)}(M) \longrightarrow \Gamma(M) \longrightarrow 1, \\
& 1 \longrightarrow P_{k}(M) \stackrel{p}{\longrightarrow} \Gamma^{k}(M) \longrightarrow \Gamma(M) \longrightarrow 1 .
\end{aligned}
$$

Hence $\Gamma^{(k)}(M) \cong \Gamma(M) \ltimes B_{k}(M)$ and $\Gamma^{k}(M) \cong \Gamma(M) \ltimes P_{k}(M)$.

Proof There is a fibration sequence

$$
\operatorname{Diff}\left(M,\left[\boldsymbol{m}_{k}\right]\right) \hookrightarrow \operatorname{Diff}(M) \stackrel{\operatorname{ev}_{\left[\boldsymbol{m}_{k}\right]}}{\longrightarrow} \Sigma_{k} \backslash \operatorname{Conf}_{k}(M) .
$$

Here $\operatorname{Diff}(M)$ is the group of (orientation-preserving) diffeomorphisms of $M$ that fix the boundary pointwise and $\mathrm{ev}_{\left[\boldsymbol{m}_{k}\right]}$ is given by evaluating a diffeomorphism at the distinguished (unordered) configuration $\left[\boldsymbol{m}_{k}\right]$. The first exact sequence in the theorem is part of the long exact sequence associated to this fibration, using the fact that $\pi_{1}(\operatorname{Diff}(M))=1$ for all surfaces $M$ with boundary; see Earle and Schatz [6, Theorem 1D]. A splitting $c: \Gamma(M) \rightarrow \Gamma^{(k)}(M)$ is given by gluing a cylinder with $k$ marked points to one of the boundary components of $M$, extending diffeomorphisms by the identity, and then smoothing. The proof in the case of the pure groups is entirely analogous.

Remark 2.6 When $M$ has genus greater than or equal to 2, the lemma above requires that $M$ have boundary. For otherwise, [7, Corollary 5.11] shows that the epimorphsm

$$
\Gamma^{1}(M) \rightarrow \Gamma(M)
$$

fails to split.

Implicit in our notation for the semidirect product is the fact that the mapping class group is acting on the right of the braid groups; the action determined by the split exact sequence is

$$
\beta \cdot \phi=p^{-1}\left(c(\phi)^{-1} \odot p(\beta) \odot c(\phi)\right)
$$

for a braid $\beta$ and a mapping class $\phi$. Here $c: \Gamma(M) \rightarrow \Gamma^{k}(M) \hookrightarrow \Gamma^{(k)}(M)$ is the splitting map introduced in the proof of Lemma 2.5. Also, our convention for the multiplication in the semidirect product is

$$
\left(\phi_{1}, \beta_{1}\right)\left(\phi_{2}, \beta_{2}\right)=\left(\phi_{1} \odot \phi_{2},\left(\beta_{1} \cdot \phi_{2}\right) \beta_{2}\right) .
$$

Another way to describe the decompositions in Lemma 2.5 is to say that (7) $\Gamma^{(k)}(M)=c(\Gamma(M)) \odot p\left(B_{k}(M)\right) \quad$ and $\quad \Gamma^{k}(M)=c(\Gamma(M)) \odot p\left(P_{k}(M)\right)$ 
and every element of the mapping class group can be written in a unique way as an element of the product. Thus, the decomposition tells us that any mapping class in $\Gamma^{(k)}(M)$ can be written in a unique way as a product $\phi_{1} \odot \phi_{2}$, where $\phi_{1}$ is fixed on a collar neighbourhood of the chosen boundary component that contains the marked points and $\phi_{2}$ is given by pushing the marked points around paths in $M$.

Let us now give a geometric description of the right action (6) of $\Gamma(M)$ on $B_{k}(M)$ that is used to form the semidirect product $\Gamma(M) \ltimes B_{k}(M)$. Consider the action of $\operatorname{Diff}(M)$ on the right of $\operatorname{Conf}_{k}(M)$ given by

$$
\left(x_{0}, \ldots, x_{k-1}\right) \cdot \phi=\left(x_{0} \cdot c(\phi), \ldots, x_{k-1} \cdot c(\phi)\right) .
$$

Recall that we think of $B_{k}(M)$ as the set of homotopy classes of paths that start at the configuration $\left(m_{0}, \ldots, m_{k-1}\right)$ and end at some permutation of it. Because $c(\phi)$ is the identity on a neighbourhood of the boundary that contains the marked points, the action just described induces an action of $\operatorname{Diff}(M)$, and subsequently of $\Gamma(M)$, on $B_{k}(M)$. We denote this action of $\Gamma(M)$ on the right of $B_{k}(M)$ by $\phi$ sending $\beta$ to $\beta^{c(\phi)}$. The notation is intended to remind us that $\beta^{c(\phi)}$ is the image of $\beta$ under the diffeomorphism $c(\phi)$.

Lemma 2.7 The action of $\Gamma(M)$ on $B_{k}(M)$ arising from the split exact sequence is the natural geometric one; that is, $p^{-1}\left(c(\phi)^{-1} \odot p(\beta) \odot c(\phi)\right)=\beta^{c(\phi)}$.

In view of the lemma, we later denote both of these quantities by $\beta \cdot \phi$.

Proof It is enough to look at the actions of $\Gamma(M)$ on the set $\mathcal{S}_{k} \cup \bigcup_{i=0}^{k-1} \mathcal{Y}_{k}^{i}$ of generators for $B_{k}(M)$. First suppose that $\beta \in \mathcal{S}_{k}$; recall from the text preceding Lemma 2.4 that the elements of $\mathcal{S}_{k}$ are represented by paths that are contained in a neighbourhood of the marked points. Thus $\beta^{c(\phi)}=\beta$ since $c(\phi)$ is the identity on a collar neighbourhood of the boundary that contains the marked points. Also, $p(\beta)$ is supported on a neighbourhood of the marked points so it commutes with $c(\phi)$ and we have that

$$
p^{-1}\left(c(\phi)^{-1} \odot p(\beta) \odot c(\phi)\right)=\beta=\beta^{c(\phi)} .
$$

Now suppose that $\beta \in \bigcup_{i=0}^{k-1} \mathcal{Y}_{k}^{i}$ so $\beta$ is represented by a path in the configuration space in which, for some $0 \leq i \leq k-1$, the first $i$ points are stationary and the remaining points move in parallel, tracing a generator $\gamma$ of $\pi_{1}\left(M-\boldsymbol{m}_{i}, m_{i}\right)$. We claim that $c(\phi)^{-1} \odot p(\beta) \odot c(\phi)=p\left(\beta^{c(\phi)}\right)$ from which it is immediate that $p^{-1}\left(c(\phi)^{-1} \odot\right.$ $p(\beta) \odot c(\phi))=\beta^{c(\phi)}$.

To see the claim, let us first assume that $\beta \in \mathcal{Y}_{k}^{k-1}=\iota_{k-1}\left(\mathcal{X}^{k-1}\right)$, say $\beta=\iota_{k-1}(\gamma)$ for $\gamma \in \mathcal{X}^{k-1}$. Thus, in the braid $\beta$, the first $k-1$ points are stationary and the last point 
traces the path $\gamma$. Hence $p(\beta)=p(\gamma)$ and for similar reasons $p\left(\gamma^{c(\phi)}\right)=p\left(\beta^{c(\phi)}\right)$. Furthermore, $p(\gamma)=\tau_{\gamma_{1}} \odot \tau_{\gamma_{2}}^{-1}$ where $\gamma_{1}$ and $\gamma_{2}$ form the boundary of a tubular neighbourhood of $\gamma$. Here we use $\tau_{\delta}$ to denote the (positive) Dehn twist around $\delta$ for a simple loop $\delta$ in $M$. For this last equality, see [7, Fact 4.7].

If $\phi: M \rightarrow M$ is a diffeomorphism and $\delta$ is a simple curve in $M$ then $\phi^{-1} \odot \tau_{\delta} \odot \phi=\tau_{\delta^{\phi}}$ (see for example [7, Fact 3.7]). Here $\delta^{\phi}$ denotes the image of the curve $\delta$ under the diffeomorphism $\phi$. Now we have that

$$
\begin{aligned}
c(\phi)^{-1} \odot p(\beta) \odot c(\phi) & =c(\phi)^{-1} \odot p(\gamma) \odot c(\phi) \\
& =c(\phi)^{-1} \odot \tau_{\gamma_{1}} \odot \tau_{\gamma_{2}}^{-1} \odot c(\phi) \\
& =\tau_{\gamma_{1}^{c(\phi)}} \odot \tau_{\gamma_{2}^{c(\phi)}}^{-1}=p\left(\gamma^{c(\phi)}\right)=p\left(\beta^{c(\phi)}\right)
\end{aligned}
$$

as claimed. In the case that $\beta \in \mathcal{Y}_{k}^{i}$ for $0 \leq i<k-1$, the diffeomorphism $p(\beta)$ is given by pushing the last $k-i$ marked points in parallel along some path $\gamma \in \mathcal{X}^{i}$. Similar reasoning shows that $c(\phi)^{-1} \odot p(\beta) \odot c(\phi)=p\left(\beta^{c(\phi)}\right)$ in this case as well.

We are now in a position to define the degeneracy maps for mapping class groups. From [2] we have degeneracy maps $s_{j}: B_{k}(M) \rightarrow B_{k+1}(M)$ given by doubling the $j^{\text {th }}$ string, and we know that these maps satisfy the simplicial identities (1). This gives us a map

$$
\operatorname{id} \ltimes s_{j}: \Gamma(M) \ltimes B_{k}(M) \longrightarrow \Gamma(M) \ltimes B_{k+1}(M)
$$

and using Lemma 2.5 we get a map $s_{j}: \Gamma^{(k)}(M) \rightarrow \Gamma^{(k+1)}(M)$. More explicitly, if we write an element of $\Gamma^{(k)}(M)$ in the form $c(\phi) \odot p(\beta)$ for $\phi \in \Gamma(M)$ and $\beta \in B_{k}(M)$ then we define

$$
s_{j}(c(\phi) \odot p(\beta))=c(\phi) \odot p\left(s_{j}(\beta)\right) .
$$

This construction restricts to give a degeneracy map on the pure mapping class group $\Gamma^{k}(M)$.

Before checking that the degeneracy maps for mapping class groups behave as desired, we prove the following lemma.

Lemma 2.8 If we define $\tilde{\pi}: \Gamma(M) \ltimes B_{k}(M) \rightarrow \Sigma_{k}$ by $\tilde{\pi}(\phi, \beta)=\pi(\beta)$ then the following diagram commutes:

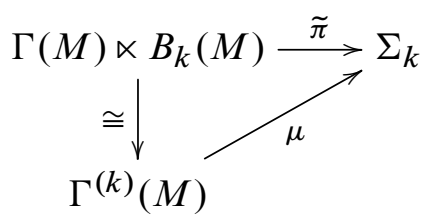


Proof The isomorphism is given by $(\phi, \beta) \mapsto c(\phi) \odot p(\beta)$, and it is evident from the definitions that $\mu(c(\phi) \odot p(\beta))=\mu(p(\beta))=\pi(\beta)=\tilde{\pi}(\phi, \beta)$.

Proposition 2.9 The degeneracy map $s_{j}: \Gamma^{(k)}(M) \rightarrow \Gamma^{(k+1)}(M)$ satisfies the crossed identity, that is, for all $\theta_{1}, \theta_{2} \in \Gamma^{(k)}(M)$ we have

$$
s_{j}\left(\theta_{1} \odot \theta_{2}\right)=s_{j}\left(\theta_{1}\right) \odot s_{j \cdot \mu}\left(\theta_{1}\right)\left(\theta_{2}\right) .
$$

Hence, the degeneracy map $s_{j}: \Gamma^{k}(M) \rightarrow \Gamma^{k+1}(M)$ is a homomorphism.

Proof By the definition of the degeneracy map and Lemma 2.8, it is enough to show that

$$
\left(\mathrm{id} \ltimes s_{j}\right)\left(\left(\phi_{1}, \beta_{1}\right)\left(\phi_{2}, \beta_{2}\right)\right)=\left(\mathrm{id} \ltimes s_{j}\right)\left(\phi_{1}, \beta_{1}\right)\left(\mathrm{id} \ltimes s_{j \cdot \pi\left(\beta_{1}\right)}\right)\left(\phi_{2}, \beta_{2}\right) .
$$

The left-hand side of (8) is

$$
\begin{aligned}
\left(\mathrm{id} \ltimes s_{j}\right)\left(\phi_{1} \odot \phi_{2},\left(\beta_{1} \cdot \phi_{2}\right) \beta_{2}\right) & =\left(\phi_{1} \odot \phi_{2}, s_{j}\left(\left(\beta_{1} \cdot \phi_{2}\right) \beta_{2}\right)\right) \\
& =\left(\phi_{1} \odot \phi_{2}, s_{j}\left(\beta_{1} \cdot \phi_{2}\right) s_{j \cdot \pi\left(\beta_{1} \cdot \phi_{2}\right)}\left(\beta_{2}\right)\right) \\
& =\left(\phi_{1} \odot \phi_{2}, s_{j}\left(\beta_{1} \cdot \phi_{2}\right) s_{j \cdot \pi\left(\beta_{1}\right)}\left(\beta_{2}\right)\right) .
\end{aligned}
$$

Here we have used the fact that the degeneracy map for the braid groups satisfies the crossed identity and the fact that for $\phi \in \Gamma(M)$ and $\beta \in B_{k}(M)$, we have that $\pi(\beta \cdot \phi)=\pi(\beta)$, which is immediate from Lemma 2.7.

The right-hand side of (8) is

$$
\left(\phi_{1}, s_{j}\left(\beta_{1}\right)\right)\left(\phi_{2}, s_{j \cdot\left(\pi\left(\beta_{1}\right)\right)}\left(\beta_{2}\right)\right)=\left(\phi_{1} \odot \phi_{2},\left(s_{j}\left(\beta_{1}\right) \cdot \phi_{2}\right) s_{j \cdot \pi\left(\beta_{1}\right)}\left(\beta_{2}\right)\right) .
$$

Thus we are reduced to checking that $s_{j}\left(\beta_{1} \cdot \phi_{2}\right)=s_{j}\left(\beta_{1}\right) \cdot \phi_{2}$; this is immediate when we consider the description of the action given in Lemma 2.7 .

We are now ready for the main theorem.

Theorem 2.10 For a surface $M$ with nonempty boundary, $\Gamma^{\mathrm{sym}}(M)$ forms a crossed simplicial group and $\underline{\Gamma}(M)$ forms a simplicial group. These simplicial structures extend the known simplicial structures for braid groups.

Proof In [4], it is shown that $\underline{\Gamma}^{\operatorname{sym}}(M)$ forms a $\Delta$-group and that the $\Delta$-group structure extends the one on $\underline{B}(M)$ that is given in [2]. We also know from Proposition 2.9 that the degeneracy maps for the mapping class groups satisfy the crossed identity, and it is immediate from the construction that they restrict to those on the braid groups. Thus, to complete the proof we just need to check that the simplicial identities hold. 
Let us define an alternative face map $\tilde{d}_{i}: \Gamma^{(k)}(M) \rightarrow \Gamma^{(k-1)}(M)$. From [2] we have face maps $d_{i}: B_{k}(M) \rightarrow B_{k-1}(M)$ given by deleting the $i^{\text {th }}$ string. This gives us a map

$$
\operatorname{id} \ltimes d_{i}: \Gamma(M) \ltimes B_{k}(M) \longrightarrow \Gamma(M) \ltimes B_{k-1}(M)
$$

and using Lemma 2.5 we get a map $\tilde{d}_{i}: \Gamma^{(k)}(M) \rightarrow \Gamma^{(k-1)}(M)$. Since the face maps and degeneracy maps for braid groups satisfy the simplicial identities, so do the maps $s_{j}$ and $\tilde{d}_{i}$ for mapping class groups. We claim that $\tilde{d}_{i}$ is the same as the face map $d_{i}: \Gamma^{(k)}(M) \rightarrow \Gamma^{(k-1)}(M)$ defined by forgetting the $i^{\text {th }}$ marked point. Given the claim, the proof that $\Gamma^{\mathrm{sym}}(M)$ is a crossed simplicial group is complete. The statement for $\underline{\Gamma}(M)$ follows by Lemma 2.1 .

To see that the claim holds, observe that

$$
\tilde{d}_{i}(c(\phi) \odot p(\beta)):=c(\phi) \odot p\left(d_{i}(\beta)\right)=c(\phi) \odot d_{i}(p(\beta))=d_{i}(c(\phi) \odot p(\beta)) .
$$

For the third equality we have used the fact that the face map for the mapping class group restricts to that for the braid group.

Observe that in the proof of Lemma 2.5 there are different choices of splitting maps corresponding to the different choices of the boundary component to which we glue the cylinder. These different splittings give rise to different semidirect product decompositions and thus different degeneracy maps. Hence the degeneracy maps are actually bi-indexed.

A relationship between the different splitting maps can be described as follows. Let $c_{i}: \Gamma(M) \rightarrow \Gamma^{(k)}(M)$ be the splitting map given by gluing a cylinder with $k$ marked points to the $i^{\text {th }}$ boundary component of $M$ and let $M(i)$ denote the surface obtained by doing this gluing.

Let $\theta: M \rightarrow M$ be a diffeomorphism that carries the $i^{\text {th }}$ boundary component to the $j^{\text {th }}$ boundary component and $\bar{\theta}: M(i) \rightarrow M(j)$ the natural extension of $\theta$. The diffeomorphism $\theta$ does not represent an element of $\Gamma(M)$ but it does represent an element of a larger mapping class group in which $\Gamma(M)$ is normal (namely the group of isotopy classes of all diffeomorphisms of $M)$. Define $\operatorname{conj}_{\theta}: \Gamma(M) \rightarrow \Gamma(M)$ by $\operatorname{conj}_{\theta}(\phi)=\theta \odot \phi \odot \theta^{-1}$. Similarly we can define $\operatorname{conj}_{\bar{\theta}}: \Gamma^{k}(M) \rightarrow \Gamma^{k}(M)$ if we think of the domain as the mapping class group of $M(j)$ and the codomain as the mapping class group of $M(i)$. 
Lemma 2.11 The splitting maps $c_{i}$ and $c_{j}$ fit into the following commutative diagram:

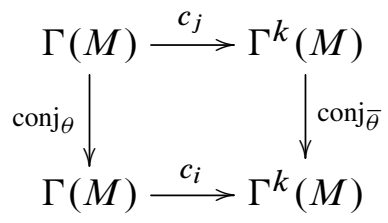

An entirely analogous result holds in the case of the full mapping class groups $\Gamma^{(k)}(M)$.

\section{Normal forms}

\subsection{Algebraic background}

Recall that if we have a split exact sequence

$$
1 \longrightarrow N \stackrel{\iota}{\longrightarrow} G \underset{s}{\stackrel{d}{\rightleftarrows}} Q \longrightarrow 1,
$$

then $G$ is isomorphic to the semidirect product $Q \ltimes N$. Alternatively, we can say that $G=s(Q) \cdot \iota(N)$ and every element of $G$ can be written in a unique way as an element of this product.

Suppose that we have two sequences of groups $N_{0}, N_{1}, \ldots$ and $G_{0}, G_{1}, \ldots$ and for each $k \geq 1$ there is a split exact sequence

$$
1 \longrightarrow N_{k-1} \stackrel{\iota_{k}}{\longrightarrow} G_{k} \underset{s_{k-1}}{\stackrel{d_{k}}{\rightleftarrows}} G_{k-1} \longrightarrow 1
$$

By iterating, we obtain that for each $k \geq 1, G_{k}$ is isomorphic to the semidirect product

$$
\left(\cdots\left(\left(G_{0} \ltimes N_{0}\right) \ltimes N_{1}\right) \ltimes \cdots\right) \ltimes N_{k-1} .
$$

Alternatively, we may express this by saying that $G_{k}$ is the product

$$
G_{k}=s_{k-1} \cdots s_{0}\left(G_{0}\right) \cdot\left(\prod_{i=1}^{k-1} s_{k-1} \cdots s_{i} \iota_{i}\left(N_{i-1}\right)\right) \cdot \iota_{k}\left(N_{k-1}\right)
$$

and every element of $G_{k}$ can be written in a unique way as an element of this product. 


\subsection{Normal forms for braid groups}

Fix a surface $M$. For each $k \geq 1$, there are short exact sequences (see for example [3, Lemma 3.11])

$$
1 \longrightarrow \pi_{1}\left(M-\boldsymbol{m}_{k}\right) \stackrel{\iota_{k}}{\longrightarrow} P_{k+1}(M) \stackrel{d_{k}}{\longrightarrow} P_{k}(M) \longrightarrow 1 .
$$

If $M$ admits a nonvanishing vector field, these sequences are split: the degeneracy map $s_{k-1}: P_{k}(M) \rightarrow P_{k+1}(M)$ provides a splitting. Thus, from the general theory in Section 3.1 we have the following.

Theorem 3.1 For every surface $M$ that admits a nonvanishing vector field and for every $k \geq 0$, there is a semidirect product decomposition

$$
P_{k+1}(M) \cong\left(\cdots\left(\left(\pi_{1}(M) \ltimes \pi_{1}\left(M-\boldsymbol{m}_{1}\right)\right) \ltimes \pi_{1}\left(M-\boldsymbol{m}_{2}\right)\right) \ltimes \cdots\right) \ltimes \pi_{1}\left(M-\boldsymbol{m}_{k}\right) .
$$

In particular, the pure braid group is a semidirect product of $k$ finitely generated free groups and $\pi_{1}(M)$.

Recall that the map $\iota_{k}$ sends a path $\gamma$ in $M-\boldsymbol{m}_{k}$ to the path in the configuration space that is constant in the first $k$ coordinates with the $(k+1)^{\text {st }}$ point tracing the path $\gamma$. The map $s_{j}$ is given by introducing a new string, close to the $j^{\text {th }}$, that moves in parallel with the $j^{\text {th }}$ string with respect to the vector field. Taking into account the behaviour of the maps $\iota_{j}$ and $s_{j}$, in the present case the expression in (9) gives Theorem B of the Introduction, as follows.

Theorem 3.2 Let $M$ be a surface admitting a nonvanishing vector field. For $k \geq 0$, every element of $P_{k+1}(M)$ can be written in a unique way in the form $\alpha_{0} \cdots \alpha_{k}$ where $\alpha_{i}$ is represented by a path in $\operatorname{Conf}_{k+1}(M)$ in which the first $i$ points are stationary and the remaining points move in parallel (with respect to the vector field), tracing a path in $M$.

Note that different decompositions and normal forms can be obtained by using different face maps and degeneracy maps in the split exact sequences (10). In particular, using $d_{0}, s_{0}$ and the map $x \mapsto\left(x, m_{1}, m_{2}, \ldots, m_{k}\right)$ yields a "left-weighted" version of the normal form in Theorem 3.2.

If the surface $M$ has nonempty boundary, an alternative splitting for the sequence (10) is provided by the map $\tilde{s}_{k-1}: P_{k}(M) \rightarrow P_{k+1}(M)$ given by $\alpha \mapsto\left(\alpha, c_{m_{k}}\right)$. Here $c_{m_{k}}$ is the map which is constant at the marked point $m_{k}$, and $m_{k}$ is considered as being close to a chosen boundary component $\partial_{0} M$. (In the terminology of [12, page 24], $\tilde{s}_{k-1}$ is the final coface map $d^{k}$.) These splittings yield another semidirect decomposition of the form in Theorem 3.1 and the following normal forms. 
Theorem 3.3 Let $M$ be a surface with boundary and $\partial_{0} M$ one of its boundary components. For $k \geq 0$, every element of $P_{k+1}(M)$ can be written in a unique way in the form $\beta_{0} \cdots \beta_{k}$, where $\beta_{i}$ is represented by a path in $\operatorname{Conf}_{k+1}(M) \subseteq M^{k+1}$ in which every coordinate is constant, except for the $i^{\text {th }}$ coordinate which follows a path in $M$ avoiding a neighbourhood of $\partial_{0} M$ that contains $m_{i+1}, \ldots, m_{k}$.

In the case of the disc, this is the well-known Artin combing of braids, as has previously been noted by $\mathrm{J} \mathrm{Wu}$, who used coface maps to obtain a bi- $\Delta$-group structure on the collection of pure braid groups $P_{k+1}(M)$. He showed [12, Proposition 1.2.9] that every bi- $\Delta$-group decomposes into iterated semidirect products in a way that parallels the arguments above.

We can also describe normal forms for elements of the full braid group. Let $\mathcal{P} \subseteq B_{n}(M)$ be a collection of braids such that $\left.\pi\right|_{\mathcal{P}}: \mathcal{P} \rightarrow \Sigma_{n}$ is a bijection.

Corollary 3.4 Any element of $B_{k}(M)$ can be written in a unique way as $\rho \alpha$ for $\alpha$ of the form given in Theorem 3.2 and $\rho \in \mathcal{P}$. Alternatively, any element can be written in a unique way as $\rho \beta$ for $\beta$ of the form given in Theorem 3.3 and $\rho \in \mathcal{P}$.

Proof This is an immediate consequence of the fact that $B_{n}(M)$ fits into an exact sequence

$$
1 \longrightarrow P_{n}(M) \longrightarrow B_{n}(M) \longrightarrow \Sigma_{n} \longrightarrow 1
$$

\subsection{Normal forms for mapping class groups}

We can use Lemma 2.5 to create semidirect product decompositions and normal forms for $\Gamma^{k+1}(M)$.

Theorem 3.5 Let $M$ be a surface with boundary. For $k \geq 0, \Gamma^{k+1}(M)$ has the semidirect product decomposition

$$
\Gamma(M) \ltimes\left(\left(\cdots\left(\left(\pi_{1}(M) \ltimes \pi_{1}\left(M-\boldsymbol{m}_{1}\right)\right) \ltimes \pi_{1}\left(M-\boldsymbol{m}_{2}\right)\right) \ltimes \cdots\right) \ltimes \pi_{1}\left(M-\boldsymbol{m}_{k}\right)\right) .
$$

Proof This is an immediate consequence of Lemma 2.5 and Theorem 3.1.

This leads to Theorem C of Section 1, as follows.

Theorem 3.6 Let $M$ be a surface with boundary. For $k \geq 0$, every element of $\Gamma^{k+1}(M)$ can be written in a unique way as $\theta \odot \phi_{0} \odot \cdots \odot \phi_{k}$, where $\phi_{i}=p\left(\alpha_{i}\right)$ for $\alpha_{i}$ as in Theorem 3.2 and $\theta$ is the identity on a collar neighbourhood of the chosen boundary component that contains the marked points.

Proof This follows from Equation (7) and Theorem 3.2. 
Theorem 3.7 Let $M$ be a surface with boundary. For $k \geq 0$, every element of $\Gamma^{k+1}(M)$ can be written in a unique way as $\theta \odot \psi_{0} \odot \cdots \odot \psi_{k}$ where $\psi_{i}=p\left(\beta_{i}\right)$ for $\beta_{i}$ as in Theorem 3.3 and $\theta$ is fixed on a collar neighbourhood of the chosen boundary component that contains the marked points.

Proof This is a consequence of Equation (7) and Theorem 3.3.

These normal forms can also be obtained independently of the results on braid groups. One considers the following exact sequences, valid for $k \geq 1$ (see [3, Lemma 3.13]):

$$
1 \longrightarrow \pi_{1}\left(M-\boldsymbol{m}_{k}\right) \stackrel{\partial^{\pi}}{\longrightarrow} \Gamma^{k+1}(M) \stackrel{d_{k}}{\longrightarrow} \Gamma^{k}(M) \longrightarrow 1 .
$$

Here $\partial^{\pi}$ is the connecting homomorphism in the long exact sequence associated to the fibration $\operatorname{Diff}\left(M, \boldsymbol{m}_{k}\right) \rightarrow M-\boldsymbol{m}_{k}$ given by evaluation at $m_{k}$. As for braid groups, there are splitting maps given either by the degeneracy $s_{k-1}: \Gamma^{k}(M) \rightarrow \Gamma^{k+1}(M)$ or by $\tilde{s}_{k-1}: \Gamma^{k}(M) \rightarrow \Gamma^{k+1}(M)$. Here $\tilde{s}_{k-1}$ is defined by gluing a cylinder with one marked point to $\partial_{0} M$ and extending diffeomorphisms by the identity, and may be considered as a final coface map, as was done for the braid groups.

We can also describe normal forms for elements of the full mapping class group $\Gamma^{(k)}(M)$. Let $\Theta \subseteq \Gamma^{(k)}(M)$ be a collection of mapping classes such that $\left.\mu\right|_{\Theta}: \Theta \rightarrow \Sigma_{k}$ is a bijection, where $\mu: \Gamma^{(k)}(M) \rightarrow \Sigma_{k}$ is as in Section 2.4 above.

Corollary 3.8 Any element of $\Gamma^{(k)}(M)$ can be written in a unique way as $\theta \odot \phi$ for $\phi$ of the form given in Theorem 3.6 and $\theta \in \Theta$. Alternatively, any element can be written in a unique way as $\theta \odot \phi^{\prime}$ for $\phi^{\prime}$ of the form given in Theorem 3.7 and $\theta \in \Theta$.

Proof This is an immediate consequence of the fact that $\Gamma^{(k)}(M)$ fits into the exact sequence

$$
1 \longrightarrow \Gamma^{k}(M) \longrightarrow \Gamma^{(k)}(M) \stackrel{\mu}{\longrightarrow} \Sigma_{k} \longrightarrow 1 .
$$

\section{References}

[1] V G Bardakov, R Mikhaillov, V V Vershinin, J Wu, On Cohen braids, preprint

[2] A J Berrick, F R Cohen, Y L Wong, J Wu, Configurations, braids, and homotopy groups, J. Amer. Math. Soc. 19 (2006) 265-326 MR2188127

[3] A J Berrick, E Hanbury, J Wu, Brunnian subgroups of mapping class groups and braid groups, Proc. Lond. Math. Soc. 107 (2013) 875-906 MR3108834

[4] A J Berrick, E Hanbury, J Wu, Delta-structures on mapping class groups and braid groups, Trans. Amer. Math. Soc. 366 (2014) 1879-1903 MR3152716 
[5] F R Cohen, On combinatorial group theory in homotopy, from: "Homotopy theory and its applications", (A Adem, R J Milgram, D C Ravenel, editors), Contemp. Math. 188, Amer. Math. Soc. (1995) 57-63 MR1349129

[6] C J Earle, A Schatz, Teichmüller theory for surfaces with boundary, J. Differential Geometry 4 (1970) 169-185 MR0277000

[7] B Farb, D Margalit, A primer on mapping class groups, Princeton Math. Series 49, Princeton Univ. Press (2012) MR2850125

[8] Z Fiedorowicz, J-L Loday, Crossed simplicial groups and their associated homology, Trans. Amer. Math. Soc. 326 (1991) 57-87 MR998125

[9] D Gonçalves, J Guaschi, Braid groups of nonorientable surfaces and the FadellNeuwirth short exact sequence, Journal Pure Applied Algebra 214 (2010) 667-677 MR2577674

[10] J Li, J Wu, Artin braid groups and homotopy groups, Proc. Lond. Math. Soc. 99 (2009) 521-556 MR2551462

[11] J Wu, A braided simplicial group, Proc. London Math. Soc. 84 (2002) 645-662 MR1888426

[12] J Wu, On maps from loop suspensions to loop spaces and the shuffle relations on the Cohen groups, Mem. Amer. Math. Soc. 851, Amer. Math. Soc. (2006) MR2203532

Yale-NUS College

6 College Avenue East \#B1-01, Singapore 138614, Singapore

Department of Mathematics, University of Durham

Science Laboratories, Durham, DH1 3LE, UK

jon.berrick@yale-nus.edu.sg, elizabeth.hanbury@durham.ac.uk

Received: 23 November 2012 Revised: 2 February 2014 